\title{
Determination of the structure and orientation of nanometer-sized precipitates in matrix materials via transmission diffraction signals emitted by bulk samples in the Scanning Electron Microscope
}

\author{
Etienne Brodu*, Suraj Dinkar Jadhav, Kim Vanmeensel, Marc Seefeldt \\ Katholieke Universiteit Leuven, Department of Materials Engineering, Heverlee, Belgium
}

* Corresponding author: Dr. Etienne Brodu, Katholieke Universiteit Leuven, Department of Materials Engineering, Kasteelpark Arenberg 44, B-3001 Heverlee, Belgium, E-mail: etienne.brodu@gmail.com, Tel: (+32) 16373228

\begin{abstract}
The characterization of precipitates in bulk materials by Electron Backscatter Diffraction becomes challenging when the precipitate size falls below $200 \mathrm{~nm}$. This is due to the drastic decrease of diffracted intensity with decreasing precipitate size and the rapidly growing signal generated by the surrounding matrix. A new technique is presented and allows determination of the structure and orientation of precipitates far below $100 \mathrm{~nm}$ directly in bulk samples in the Scanning Electron Microscope, normally requiring thin lamella extraction and Transmission Electron Microscopy analysis. The technique relies on the selective chemical etching of the matrix material of a sample, combined with the large tilt characteristic of the EBSD technique. As a result of the selective etching, precipitates protrude from the surface and emit what turns out to be a transmission diffraction signal instead of a backscatter diffraction signal, even though the sample remains massive. With this technique successful analysis of precipitates down to $30 \mathrm{~nm}$ was performed in a bulk $\mathrm{Cu} / \mathrm{Cr}$ sample produced by Laser Powder Bed Fusion Additive Manufacturing with standard EBSD hardware. A Monte Carlo simulation confirms that the intensity of the transmission signal collected from a nanometer-sized precipitate protruding from the surface is much higher than the intensity of the backscatter signal collected from the same precipitate embedded right underneath the surface of a flat EBSD sample. Transmission signals emitted by bulk samples are a great opportunity to study features whose size are below the resolution limit of EBSD, like nanometer-sized precipitates, while keeping the possibility of exploring large areas and without the need to produce a thin lamella.
\end{abstract}


Keywords: Precipitation strengthening; Nanometer-sized precipitates; Orientation relationship; Electron Backscatter Diffraction (EBSD); Transmission Kikuchi Diffraction (TKD); Scanning Electron Microscope (SEM)

\section{Introduction}

Precipitation strengthening of materials often provides the best results in terms of yield strength when precipitates are coherent or semi-coherent, because the strain fields induced by the lattice mismatch between precipitate and matrix and surrounding the precipitates limit the motion of dislocations. This effect is referred to as coherency strengthening. Large precipitates on the contrary are usually incoherent because they nucleate interfacial dislocations as they grow, resulting in a progressive loss of coherency and coherency strengthening [Shi 2012]. With increasing precipitate size, when evolving from an under-aged towards an over-aged alloy, Orowan looping replaces progressively precipitate cutting as the predominant interaction with dislocations. Hence the final outcome in terms of mechanical properties for a given alloy strongly depends on the structure, coherency and size of precipitates, with a maximum strength often being achieved for a specific combination of these parameters, as indicated by the socalled one-peak age hardening curve [Guo 2002].

Current techniques that allow to determine the structure and orientation of precipitates smaller than $100 \mathrm{~nm}$ are rare and are mostly based on Transmission Electron Microscopy (TEM). TEM techniques allow structure, orientation and even chemical analysis (including core-shell structures), with seemingly no limitation in terms of precipitate size. Besides, the coherency of the interface can be analyzed in detail either via a misfit strain analysis [Song 2013] or by analysis of misfit defects [Fuji 2000, Liu 2018]. In comparison, the analysis capabilities of Scanning Electron Microscopes are very limited. A recent technique derived from Electron Backscatter Diffraction (EBSD), Transmission Kikuchi Diffraction (TKD), allows nanoscale orientation mapping in SEM and contributes to reducing the gap with TEM capabilities [Sneddon 2016]. It has been shown that TKD does allow characterization of precipitates below $100 \mathrm{~nm}$ [Suzuki 2013, Liu 2019], but their analysis remains very challenging because of the strong depth selectivity of the TKD technique. Indeed, unlike spot diffraction, a small amount of matrix material, with a thickness of 10-20 nm and located between a precipitate and the back surface of a lamella, can be sufficient to make the contribution of a precipitate to the Kikuchi pattern marginal by absorption, depending on atomic number and 
incident energy [Brodu 2017-1, Liu 2019]. As a consequence, the matrix material often remains the main, if not the only contributor to the collected diffraction patterns.

Using the replica technique, it is possible to overcome the problem of the overwhelming contribution from the matrix material to diffraction patterns. Bhattacharya et al. [Bhattacharya 2019] used the replica technique, consisting in replacing the native matrix material by carbon via etching/deposition steps, in combination with TKD, and reports characterization of precipitates down to $20 \mathrm{~nm}$. The major advantage of the replica technique is that it allows highly automated data collection and provides with great statistics. The major disadvantage is that the precipitate-matrix orientation relationship is lost as soon as the matrix is removed. Similarly, diffraction analysis of particles in the range 5-10 $\mathrm{nm}$ was previously reported, either with no matrix around or in a light matrix (e.g. Pb-Sn particles in carbon) [Keller 2012, Brodusch 2013]. Besides diffraction, chemical EDX analysis in SEM of particles down to 20 $\mathrm{nm}$ deposited on electron-transparent foils has also been reported by Hodoroaba [Hodoroaba 2016]. Alternatively, Atom Probe Tomography (APT) is also a powerful technique that allows determining the chemical composition and morphology of nanometer-sized precipitates [Jiao 2017, Chbihi 2012], with a resolution close to the nanometer [Breen 2014].

The new SEM technique that we propose is based on the use of transmission diffraction signals emitted by bulk samples and allows direct diffraction analysis of nanometer-sized precipitates in bulk samples with standard EBSD hardware. Contrary to the replica technique, orientation relationships are preserved, and contrary to the use of thin foils, very large fields of view can be explored. The second section of this study will discuss precipitate imaging modalities. The third section details the acquisition and post-treatment routes that we used for the determination of the structure and orientation of precipitates. The fourth section summarizes and discusses the results of the precipitate characterization for a $\mathrm{Cu} / \mathrm{Cr}$ sample produced by Laser Powder Bed Fusion Additive Manufacturing. The fifth section discusses the physics behind the technique, illustrated and quantified via Monte Carlo simulations of the electron-matter interaction. Finally, the last section proposes a comparison with conventional EBSD.

\section{Material elaboration and sample preparation}

The current study aims at the determination of the structure and orientation of nanometer-sized precipitates in a metallic matrix using transmission diffraction signals emitted 
by bulk samples in the Scanning Electron Microscope. As a model system, a $\mathrm{Cu}$ alloy, reinforced with $\mathrm{Cr}$ and $\mathrm{CrN}$ precipitates, was produced by Laser Powder Bed Fusion (LPBF) Additive Manufacturing (AM). Copper-chromium samples were produced from a modified $\mathrm{CuCr} 1$ powder presenting a chromium and chromium-nitride enriched surface layer. The LPBF process generates a supersaturated solid solution of chromium and nitrogen in the copper matrix because of the high cooling rate $\left(\approx 10^{4}-10^{6} \mathrm{~K} / \mathrm{s}\right)$, resulting in a low electrical and thermal conductivity. For this reason, a direct age hardening (DAH) post-treatment at $450^{\circ} \mathrm{C}$ for $9 \mathrm{~h}$ was applied to the fabricated parts in order to enhance the electrical and thermal conductivity as well as the mechanical properties, by triggering the precipitation of $\mathrm{Cr}$ and/or $\mathrm{CrN}$ in the $\mathrm{Cu}$ matrix. Two specific goals are aimed at: a) characterization of the chemical composition of the precipitates, which more specifically involves determining whether they are metallic $\mathrm{Cr}$ or ceramic $\mathrm{CrN}, \mathrm{b}$ ) determining whether they exhibit a coherency relationship with the $\mathrm{Cu}$ matrix, based on the presence or not of specific orientation relationships. Both the chemical nature and the absence/presence of crystallographic coherency determine the interaction of precipitates with dislocations and ultimately the mechanical properties.

Bulk samples were first ground with papers up to 4000-grit. They were then polished manually with a water based $3-\mu \mathrm{m}$ diamond solution on a napped cloth. Finally, they were etched by immersion for 10 seconds in a solution commonly used for the preparation of copper alloys samples and composed of iron(III) chloride (3.5 g), hydrochloric acid (2.5 ml), and ethanol $(75 \mathrm{ml})$ [Handbook], followed by a cleaning step under a stream of water and then in ethanol in an ultrasonic bath for $30 \mathrm{~min}$. This chemical solution selectively etches the copper matrix and leaves the chromium precipitates intact. As a result, the precipitates protrude from the sample surface. This is the key to the success of the present technique. A strong surface topography is also generated in the copper matrix because of the crystal orientation dependence of the etching speed. A second batch of samples of the same material was prepared in order to conduct conventional EBSD analysis. In the latter case, the same sample preparation protocol was used, except that the last step, i.e. the chemical etch, was replaced by a 2 minutes OPS polish. Unlike the etched samples, the samples prepared in this way present no surface topography and are flat. The diffraction patterns were recorded with an Hikari Super camera from EDAX in a FEI Nova nanoSEM 450 Scanning Electron Microscope, and were then indexed with the TSL-OIM software. Additional methodological details are provided all along the text. 


\section{Imaging precipitates}

While the next sections focus on the help provided by the chemically-induced surface topography for diffraction analysis, this section focuses on the possibility to locate and image nanometer-sized precipitates. Precipitates protruding from the surface exhibit good contrast in Secondary Electron (SE) images because these electrons are highly sensitive to variations of the local inclination angle (type $1 \mathrm{SE}$ in particular). The so-called edge effect of secondary electrons highlights even further the contour of precipitates thanks to a local increase of brightness at precipitate edges (fig. 1), which is very convenient for determining their shape and size. The TLD detector ("Through-lens" SE detector) that we used collects both type 1 and type 2 secondary electrons simultaneously, i.e. electrons generated by the primary interaction with the sample and generated by backscatter electrons on their way out of the sample, respectively. A great advantage of the TLD detector is that, unlike an ETD detector (EverhartThornley SE detector) it collects electrons symmetrically around the incident direction. As a result, the often misleading asymmetric topographic shadowing is suppressed in images. For the sake of comparison, imaging of the precipitates of the same material was also performed with a flat sample (i.e. conventionally prepared EBSD sample). The same TLD detector was used for imaging. According to figure 1, analysis of the shape and size of precipitates seems also possible in flat samples via the atomic number contrast of secondary electrons (or alternatively with Backscattered Electrons with a BSE detector, like in [Jozwik 2018]), but overall the resolution and contrast are not as good as when precipitates sit on the surface. In particular, the contour of precipitates is often both blurred and poorly contrasted. The poor contrast of precipitate contours might result in part from the decreasing projected thickness of precipitates with increasing distance from precipitate centers. This effect is visible with the largest precipitate in fig. 1, which shows a grey level variation when moving away from its center and in particular a lighter top-right section. According to the BSE images presented in [Jozwik 2018], we can assume that a second effect affects both the contrast and sharpness of precipitate contours. Although this is not commented in [Jozwik 2018], images are striking and simultaneously show, within the same field of view, highly blurred/poorly contrasted precipitates or very sharp/highly contrasted precipitates for a constant precipitate size. Our guess is that this effect is related to the in-depth position of precipitates under the surface. Finally, with the flat sample, an additional blur is also likely induced by the larger interaction volume of the type $2 \mathrm{Z}$-contrast secondary electrons, in comparison to the type 1 topographiccontrast secondary electrons responsible for the contrast in the etched sample. For all these 
reasons, imaging nanometer-sized precipitates appear easier and more accurate after a selective chemical etch of the matrix.

According to $\mathrm{SE}$ images of the etched $\mathrm{Cu} / \mathrm{Cr}$ sample, the large majority of precipitates range from 20 to $100 \mathrm{~nm}$ in size, with a small fraction in the 100 to $200 \mathrm{~nm}$ range. In terms of morphology, they do present some facets but are overall spherical/ellipsoidal and not plateshaped (see [Hall 1972, Luo 1994, Luo 1998] for examples of plate-shape Cr precipitate). A second population of even smaller precipitates $(<10 \mathrm{~nm})$ is present in the sample and can be seen in the background of the SE images as small dots in figure 1 (white and black in the left and right images, respectively). These precipitates are not treated in this study as they are out of our reach for the SEM technique that we propose.
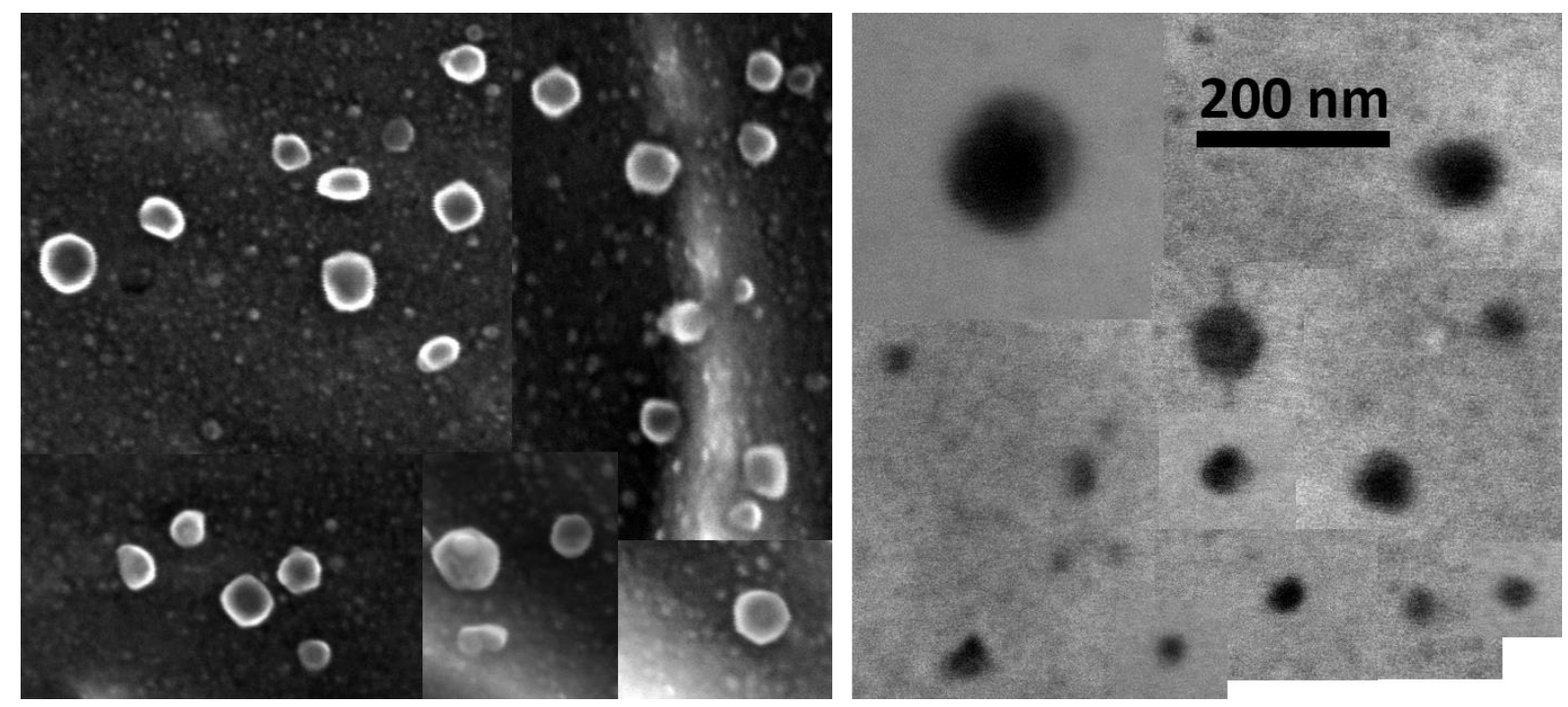

(one column figure) Figure 1: Secondary electron images (10 keV, immersion mode, TLD detector, no sample tilt) of $\mathrm{Cr}$ precipitates in a $\mathrm{Cu}$ matrix for two different surface preparation routes. Left: after surface polishing, the $\mathrm{Cu}$ matrix was selectively etched. As a result, $\mathrm{Cr}$ precipitates protrude from the sample surface. Right: the surface was conventionally prepared for EBSD analysis, with an OPS finish. Hence, in this second case, the surface is flat and precipitates remain embedded in/under the surface.

\section{Diffraction pattern acquisition and post-processing}

This section details the acquisition and post-processing routes that were used to retrieve the structure and orientation of precipitates as small as $30 \mathrm{~nm}$ directly from bulk samples with a standard EBSD detector. While the next section will discuss the physics behind the technique, this section only details the methodology associated with the technique. The sample was first tilted to $60^{\circ}$ in order to record EBSD diffraction patterns. While a tilt of $70^{\circ}$ is commonly 
preferred for EBSD mapping since it maximizes the signal intensity on the scintillator, it was observed that images of a rough surface produced by secondary electrons are much easier to interpret at lower tilt angles, which in turns makes the diffraction analysis of precipitates easier. The electron energy was set to $15 \mathrm{keV}$ in order to obtain the highest possible contribution from precipitates to diffraction patterns. At higher energy, the incident electrons are more likely to reach the matrix material that is located underneath/around the precipitates, which would result in an increased contribution from the matrix to the recorded diffraction patterns. Obviously, it would be tempting to decrease even further the energy. However, this often results in much poorer diffraction patterns. Indeed, at $10 \mathrm{keV}$, Kikuchi bands become very broad and are consequently harder to detect and index, in particular if the detection is done via an automatic Hough algorithm. Besides, at $10 \mathrm{keV}$ as soon as the surface presents either an amorphous layer (typically induced by Focus Ion Beam milling), a severely deformed layer (from mechanical polishing), or an oxide/contamination layer, then the band contrast is much lower than at 15 $\mathrm{keV}$ or $20 \mathrm{keV}$ because the characteristic absorption length of elastic waves (including Kikuchi diffraction) is linearly decreasing with decreasing energy [Brodu 2017-1]. Finally, the conversion efficiency of current EBSD detectors decreases with decreasing energy, with a threshold often around $5 \mathrm{keV}$. Since we need only one pattern from a given precipitate and only one pattern from the matrix nearby in order to retrieve both structures as well as the orientation relationship, the acquisition of diffraction patterns was done in "spot mode", meaning that the incident beam was manually set at a fixed location, either on a precipitate or on the matrix, with the help of an SE image (see figure 2). The reason to work in spot mode is also to acquire patterns with high signal/noise ratio via the use of long acquisition times combined with image averaging (200 ms $\times 5$ images for the patterns in figure 2). Again, it is easy to set the incident beam right on a given precipitate because they are easy to locate with secondary electrons. As an alternative, it could also be convenient to record the diffraction patterns in mapping mode on small areas containing precipitates. However, it would require to locate the diffraction patterns associated to precipitates within the pattern dataset afterwards, because a posttreatment of these patterns is necessary. Indeed, even though the present technique allows a drastic increase of precipitate-to-matrix signal ratio in diffraction patterns, a contribution from the matrix material often remains present in precipitate patterns, and needs to be removed in order to achieve a high indexing confidence. A metric like the quality of diffraction patterns (often referred to as "quality map") could be a way of finding these patterns. However, even with the help of the quality map, finding the patterns associated to the precipitates in the dataset might be challenging if the matrix topography and defect density is high, as is the case for the 
present sample, because the quality map would be hard to read to the naked eye as well as to process with an image analysis software. This is why we opted for the spot mode. In this study the post-treatment of precipitate patterns involved subtracting a matrix pattern from the pattern recorded with the beam incident on a precipitate (see figure 2). The matrix pattern used for subtraction was also recorded in spot mode, right next to the precipitate. Finally, once a set of two patterns was obtained, one containing information from the precipitate (i.e. after subtraction of the matrix) and one with information from the matrix, the structures and the orientation relationship could be retrieved. Indexing of both precipitate and matrix patterns was done manually because Hough detection algorithms tend to mistake Kikuchi lines for Kikuchi bands, especially with high order planes, which results in systematic errors up to a few degrees. This type of error is not affecting intra-granular misorientation profiles because the error tends to be constant for a given orientation range (i.e. the software makes always the same mistake as long as the pattern remains similar). In opposition, in the case of two neighboring grains or a precipitate in a matrix, the orientation relationship can be erroneous by several degrees because the systematic errors associated to each crystal can be largely different.

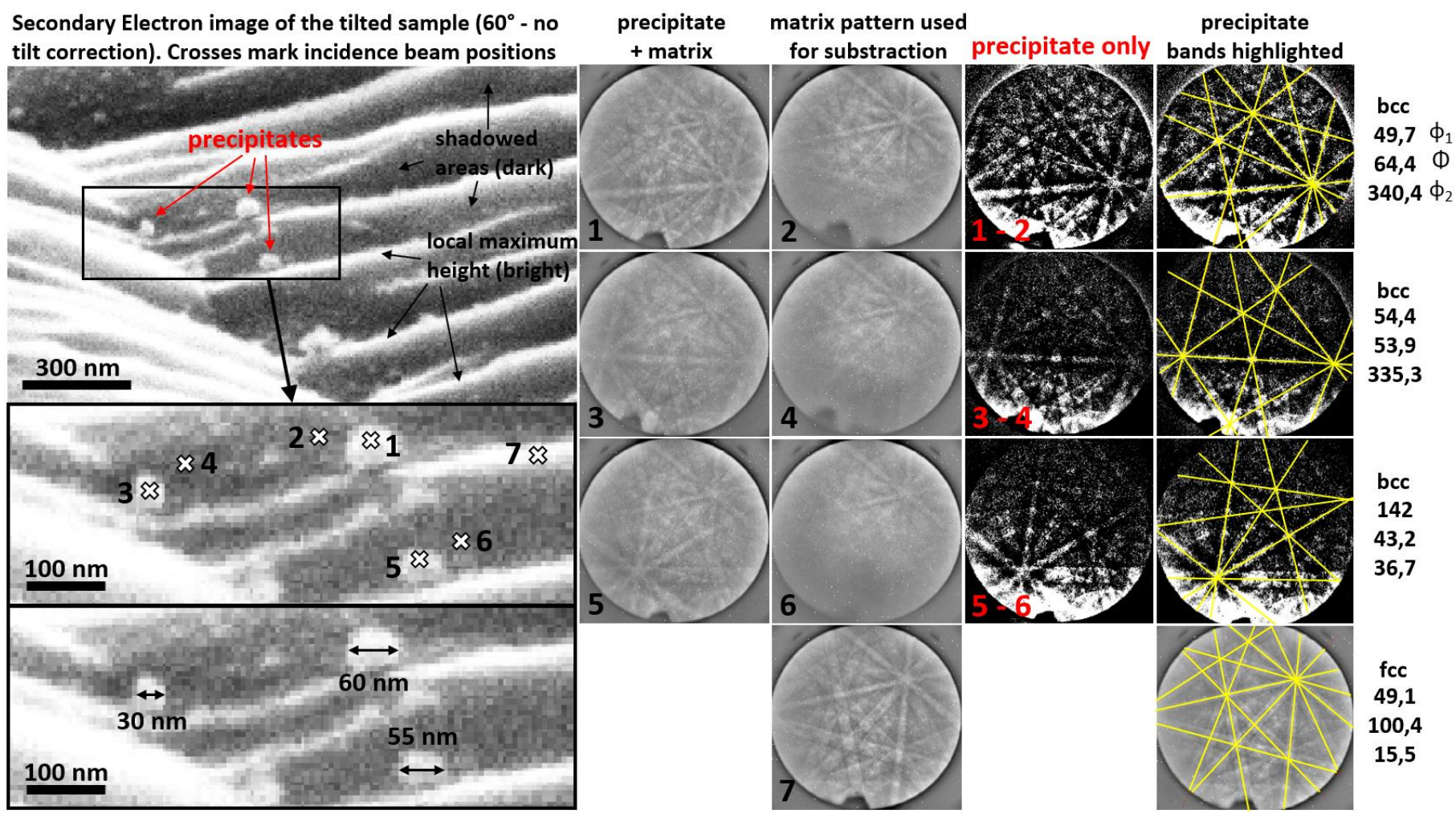

(two columns figure, color) Figure 2: Determination of the structure and orientation of three nanometersized precipitates in a bulk $\mathrm{Cu}-\mathrm{Cr}$ sample with conventional EBSD hardware in SEM. After surface polishing, the surface of the bulk sample was chemically etched, resulting in precipitates protruding from the surface. The surface is tilted by $60^{\circ}$ and the incident energy is $15 \mathrm{keV}$. 


\section{Results of the $\mathrm{Cr}$ precipitate characterization}

In total, seven precipitates ranging from 30 to $70 \mathrm{~nm}$ were analyzed with this technique in the $\mathrm{Cu} / \mathrm{Cr}$ etched sample. The results are summarized in table 1 . First, based on the bcc structure identified, this analysis reveals that the precipitates are metallic $\mathrm{Cr}$ and not $\mathrm{Cr}$ nitrides. For the same reason, coherent fcc precipitates are also ruled out. Then, in terms or orientation relationship (OR) with the fcc $\mathrm{Cu}$ matrix, based on the misorientation axis [Verbeken 2009], three are close to the Nishiyama-Wassermann (NW) OR, as already reported for the $\mathrm{Cu} / \mathrm{Cr}$ system [Hall 1972, Luo 1994, Gautam 2013], one is close to the Bain OR, which was not reported until now for the $\mathrm{Cu} / \mathrm{Cr}$ system to our knowledge, and finally three are significantly away from any known OR for the fcc/bcc system (see [Nolze 2004] and [Verbeken 2009] for an overview of the fcc/bcc ORs). According to [Fuji 2000] the Kurdjumov-Sachs (KS) OR becomes the dominant $\mathrm{OR}$ with increasing ageing time for the $\mathrm{Cu} / \mathrm{Cr}$ system. However, this OR was not observed in the present study, which is consistent with the fact that we observed a spherical/ellipsoidal precipitate morphology (with some facets), while $\mathrm{Cr}$ precipitates that satisfy the KS-OR tend to be plate-shaped [Chbihi 2012]. It also suggests that the direct age hardening process parameters that we used $\left(450^{\circ} \mathrm{C}\right.$ for $\left.9 \mathrm{~h}\right)$ stopped the process at an intermediate state. These ORs suggest some degree of coherency of the $\mathrm{Cr}$ precipitates with the $\mathrm{Cu}$ matrix, for at least a significant part of the precipitates. In order to build the table 1 , for each doublet of precipitate/matrix experimental euler angles, it first consisted in calculating the matrix/precipitate misorientation axis and angle. This misorientation axis is then expressed in the precipitate crystal reference frame, as reported in table 1 , and is finally compared to the known fcc/bcc OR misorientation axis that is the closest. The difference is reported in the last column as the angle between the experimental and known misorientation axis and is within a few degrees for 4 precipitates out of 7 . 


\begin{tabular}{|c|c|c|c|c|c|c|c|c|c|c|c|c|}
\hline & \multicolumn{3}{|c|}{ experimental euler angles } & $\begin{array}{l}\text { precipitate } \\
\text { size }(\mathrm{nm})\end{array}$ & \multicolumn{4}{|c|}{ experimental orientation relationship (OR) } & \multicolumn{3}{|c|}{ closest known fcc/bcc OR } & $\begin{array}{c}\text { angle }\left({ }^{\circ}\right) \\
\text { between (1) } \\
\text { and (2) }\end{array}$ \\
\hline $\begin{array}{c}\text { matrix } \\
\text { precipitate }\end{array}$ & $\begin{array}{c}51,0 \\
124,2\end{array}$ & $\begin{array}{l}100,2 \\
82,1\end{array}$ & $\begin{array}{l}26,5 \\
44,9\end{array}$ & $\begin{array}{l}/ \\
50\end{array}$ & 36,7 & 0,79 & 0,59 & 0,17 & / & / & / & / \\
\hline $\begin{array}{c}\text { matrix } \\
\text { precipitate }\end{array}$ & $\begin{array}{c}50,9 \\
353,8\end{array}$ & $\begin{array}{c}100,2 \\
63,3\end{array}$ & $\begin{array}{l}26,5 \\
73,1\end{array}$ & $\begin{array}{c}/ \\
55\end{array}$ & 39 & 0,89 & 0,38 & 0,24 & I & / & / & / \\
\hline $\begin{array}{c}\text { matrix } \\
\text { precipitate }\end{array}$ & $\begin{array}{r}291,7 \\
44,4\end{array}$ & $\begin{array}{l}98,2 \\
84,7\end{array}$ & $\begin{array}{c}294,5 \\
50,3\end{array}$ & $\begin{array}{l}/ \\
70\end{array}$ & 52 & 0,96 & 0,25 & 0,07 & 0,98 & 0,2 & 0,08 & 3 \\
\hline $\begin{array}{c}\text { matrix } \\
\text { precipitate }\end{array}$ & $\begin{array}{l}49,1 \\
49,7\end{array}$ & $\begin{array}{l}100,4 \\
64,4\end{array}$ & $\begin{array}{c}15,5 \\
340,4\end{array}$ & $\begin{array}{l}/ \\
60\end{array}$ & 49,8 & 0,99 & 0,08 & 0,06 & 1 & 0 & 0 & 6 \\
\hline $\begin{array}{c}\text { matrix } \\
\text { precipitate }\end{array}$ & $\begin{array}{c}49,1 \\
142,0\end{array}$ & $\begin{array}{c}100,4 \\
43,2\end{array}$ & $\begin{array}{l}15,5 \\
36,7\end{array}$ & $\begin{array}{c}/ \\
55\end{array}$ & 42,8 & 0,95 & 0,28 & 0,1 & 0,98 & 0,2 & 0,08 & 5 \\
\hline $\begin{array}{c}\text { matrix } \\
\text { precipitate }\end{array}$ & $\begin{array}{l}49,1 \\
54,4\end{array}$ & $\begin{array}{c}100,4 \\
53,9\end{array}$ & $\begin{array}{r}15,5 \\
335,3\end{array}$ & $\begin{array}{l}/ \\
30\end{array}$ & 53,6 & 0,84 & 0,36 & 0,40 & / & / & / & / \\
\hline $\begin{array}{c}\text { matrix } \\
\text { precipitate }\end{array}$ & $\begin{array}{l}84,7 \\
18,1\end{array}$ & $\begin{array}{l}37,5 \\
42,7\end{array}$ & $\begin{array}{r}0,6 \\
11,5\end{array}$ & $\begin{array}{c}/ \\
45\end{array}$ & 58,84 & 0,99 & 0,15 & 0 & 0,98 & 0,2 & 0,08 & 5 \\
\hline
\end{tabular}

same matrix grain

Nishiyama-Wasserman OR

Table 1: Summary of the experimental euler angles for seven bcc $\mathrm{Cr}$ precipitates and the nearby fcc $\mathrm{Cu}$ matrix and analysis of the precipitate/matrix orientation relationships.

\section{The physics behind the technique}

After a closer look at the diffraction patterns, it was very intriguing that the contributions from precipitates were always predominantly located in the bottom sections of diffraction patterns while the matrix was mostly present in the top sections. For example, we can see in figure 2 that the brightness and signal/noise ratio of Kikuchi bands is higher in the bottom sections of "precipitate only" patterns, with the very bottom sections even being saturated. Monte Carlo simulations of electron trajectories conducted with the CASINO software [Drouin 2007] provide an explanation for this behavior and also explain how it is possible to obtain indexable diffraction signals from such tiny precipitates in bulk samples. A simulation that reproduces our experimental set-up was created and run, which includes the detector geometry (pattern center, opening angle) and the microscope parameters (energy, beam size, WD) (see the caption of figure 3 for more details). The sample was simulated as a $50 \mathrm{~nm}$ cubic precipitate lying on the surface of a bulk sample tilted by $60^{\circ}$. Then the landing positions of electrons on the EBSD detector as a function of the material from which they escape, either the precipitate or the matrix, were extracted from the simulation. As can be seen from figure 3 , the simulation reproduces the experimental behavior: the electrons emitted by 
the precipitate and reaching the detector are predominantly located in the bottom section of the detector while the matrix electrons tend to preferentially occupy the top section. Hence, the angular distribution of the intensity of the signal emitted by the precipitate presents a maximum in the direction of the incident direction and a decreasing intensity with increasing scattering angle (i.e. towards the top of the detector), which is typical of a transmission signal. The same angular behavior is obtained with the Transmission Kikuchi Diffraction technique performed on thin lamella [Brodu 2017-2], and simply finds its origin in the forward direction of the electron-nucleus interaction cross-section. In opposition, the backscatter signal emitted by the matrix presents a peak intensity at intermediate angles on the detector. In order to support the claim that the signal generated by a protruding precipitate is indeed a transmission signal, an additional simulation was conducted and the results are shown in figure 4 . One can see that the signal generated by a free-standing precipitate, with no matrix material underneath, has the exact same intensity and angular distribution on the detector as the one generated by the protruding precipitate, as if the matrix material of the bulk sample did not play any role in the precipitate signal generation. This is consistent with the fact that according to the simulation, 98\% of the electrons escaping from the $50 \mathrm{~nm} \mathrm{Cr}$ protruding precipitate have travel paths strictly located within the precipitate and have no contact whatsoever with the $\mathrm{Cu}$ matrix along their paths before they escape the sample. In this scenario we could almost see the precipitate as a thin lamella with no lateral elongation (cubic in the simulation or of various shape in the real world) supported by a bulk sample almost acting as a Focus Ion Beam thin lamella holder, except that this holder does also produce some signal, but of the traditional backscatter type in opposition to the precipitate. This configuration allows the generation of an intense precipitate signal: a protruding precipitate as little as $50 \mathrm{~nm}$ generates a precipitate-to-matrix signal ratio on the detector as high as $40 \%$ (section 5 will show that precipitates in conventional EBSD generate much less signal). This is the reason why signals from such tiny precipitates in a bulk sample can be indexed. As can be seen in figure 3, the signal from a nanometer-sized precipitate even tends to be predominant over the matrix signal in the bottom section of the detector.

Then, it is possible to further increase the ratio of precipitate-to-matrix signal in diffraction patterns by selecting specifically precipitates located on top of local maximum heights on the surface. By doing so, the contribution from the matrix tends to be reduced by shadowing because the matrix signal is produced downhill. This was used for the analysis of the three precipitates presented in figure 2, where we can see that the contributions of the matrix to the patterns is very limited. By doing this, we do not increase the signal from precipitates 
though; only the precipitate-to-matrix signal ratio is increased, but it makes the processing of the precipitate pattern easier and is also a good way to make sure that the precipitate signal is not reduced by shadowing. In order to complete the data set and retrieve the orientation relationship, a matrix pattern was also recorded for an incident beam position set on a local maximum height (pattern 7 in figure 2), in order to facilitate indexing of the matrix material. Areas of local maximum heights are easily identified with secondary electrons and correspond to bright areas in images. 

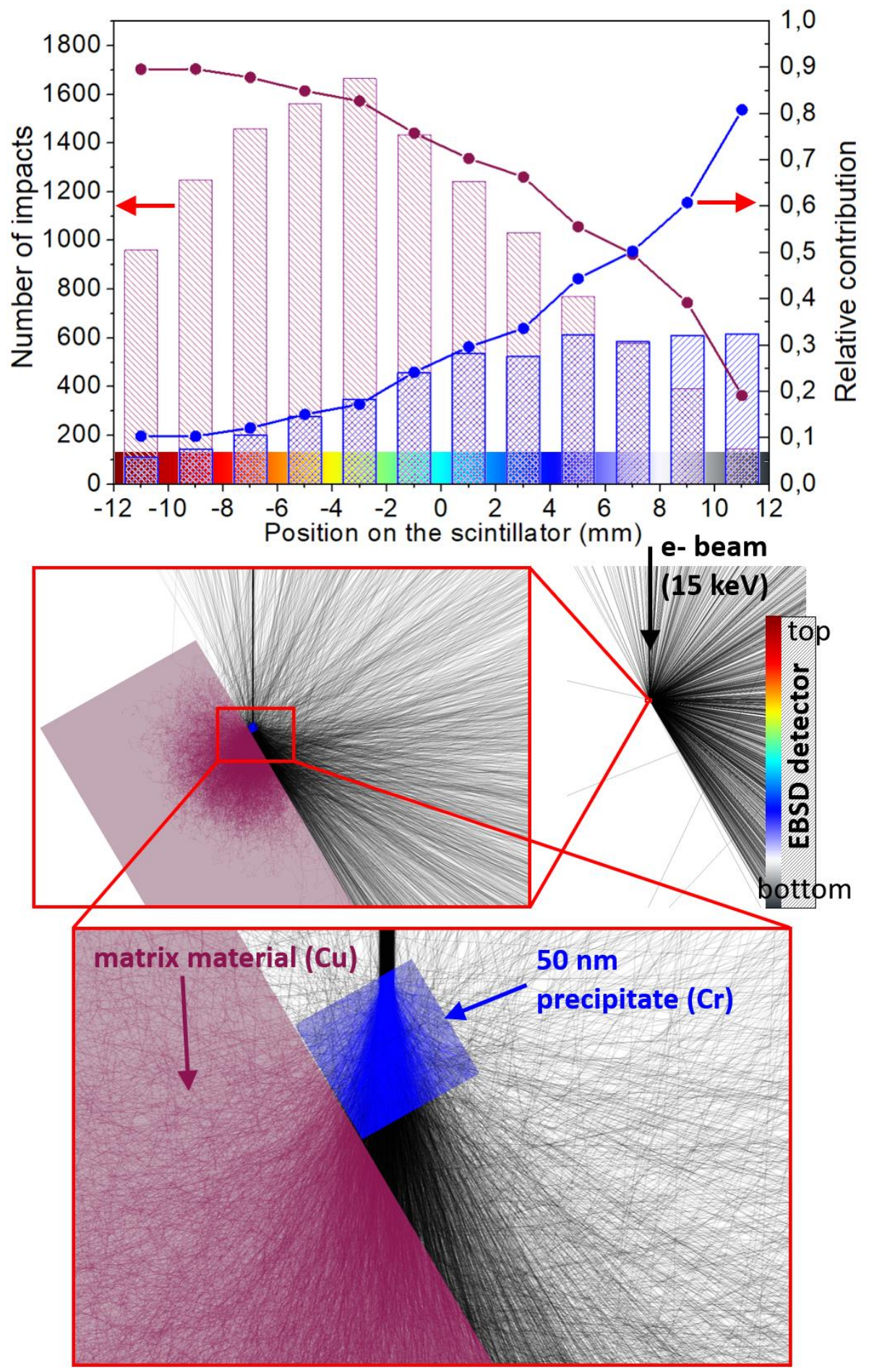

(one column figure, color) Figure 3: Result of a Monte Carlo simulation (CASINO [Drouin 2007]) showing the landing position of electrons and their relative intensity on an EBSD detector as a function of the material from which they escape, either the precipitate in blue or the matrix in purple. The precipitate protrudes from the surface and generates a signal that is in part in transmission. The WD is $11 \mathrm{~mm}$, the sample tilt is $60^{\circ}$, the detector opening angle is $68^{\circ}$ and the projection of the pattern center is located at one fourth from the top on the scintillator. The $24 \mathrm{~mm}$ scintillator was scaled down in the simulation to ease calculations. Still, all angles remain the same as in the experiment of figure 2. Energy is $15 \mathrm{keV}$, probe size is $5 \mathrm{~nm}, 50000$ trajectories simulated, 2000 displayed. 

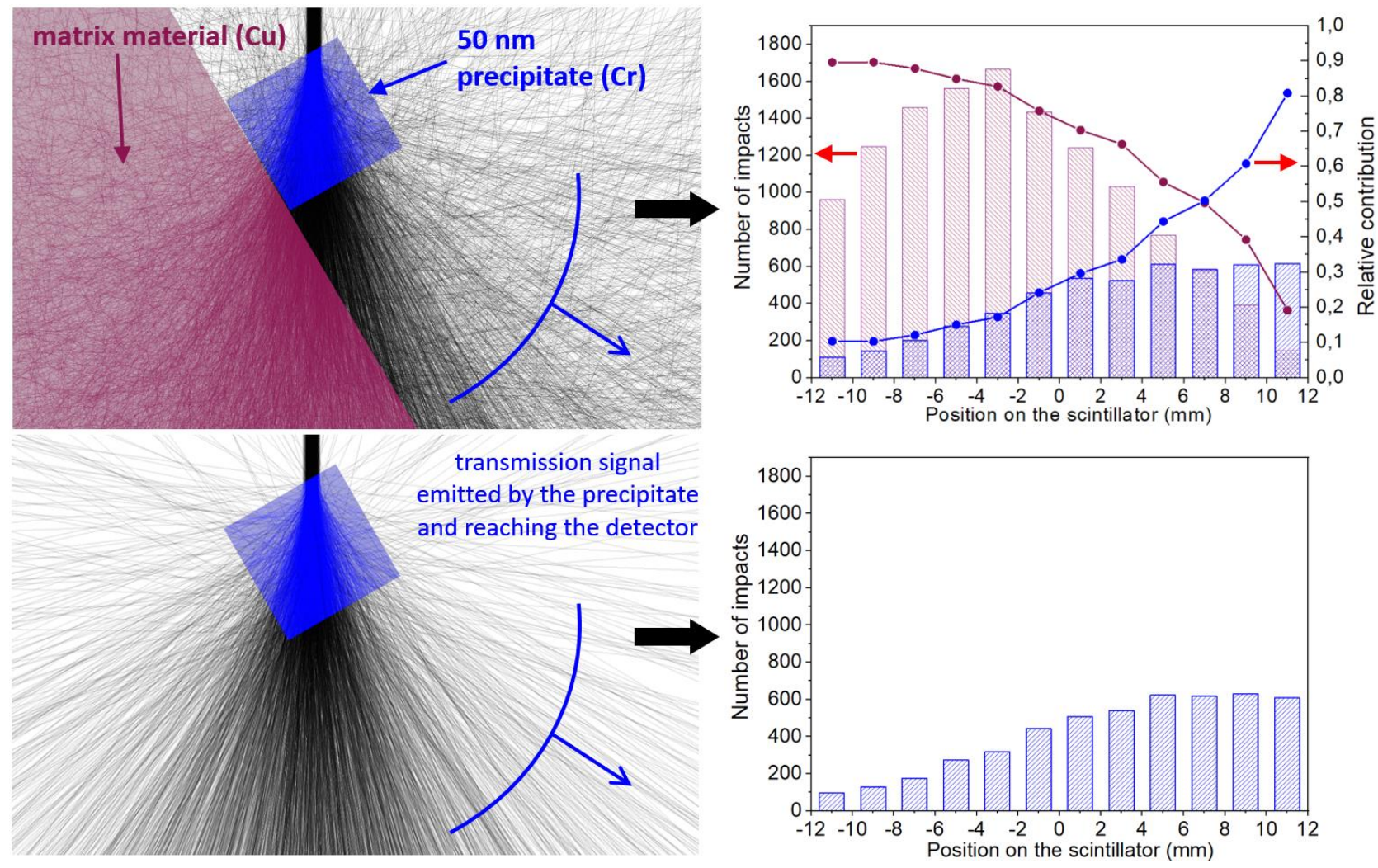

(one column figure, color) Figure 4: Result of a Monte Carlo simulation (CASINO [Drouin 2007]) showing the landing position of electrons and their relative intensity on an EBSD detector as a function of the material from which they escape, either the precipitate in blue or the matrix in purple. Two scenarios are compared: (top) a precipitate protruding from a bulk sample (same as figure 3) and (bottom) a free-standing precipitate with no supporting bulk sample. All parameters of the simulation are the same as in figure 3.

\section{Comparison with a conventional, flat EBSD sample}

A second simulation was then carried out with the objective of evaluating the gain in signal intensity obtained when a precipitate protrudes from the surface in comparison to a precipitate that is embedded underneath the surface of a flat EBSD sample. The same $50 \mathrm{~nm}$ $\mathrm{Cr}$ precipitate, as used in the previous section, was used for this simulation, except that the precipitate is now positioned right below the surface of the sample, i.e. one of its faces coincides with the surface of the bulk sample, as shown in Figure 5. We can expect that the diffraction signal emitted by such precipitate largely depends on the incident beam position on the sample surface, and that the maximum signal intensity is likely not obtained with a beam directly incident on the precipitate. For this reason, the first step of the simulation consisted in locating the position of highest backscatter emission density. Then, for a fair comparison with the etched sample, the precipitate was purposely set at this location. Finally, similarly to the previous section, the landing positions of electrons on the EBSD detector as a function of the 
material from which they escape, either the matrix or the precipitate, were extracted from the simulation. The results are presented in figure 5.

According to figure 5, the first outcome of that simulation is that the intensity variation with scattering angle of the signal emitted by the precipitate is not anymore the one of a transmitted signal but is instead the one of a conventional backscatter signal, with an angular distribution similar to the one of the surrounding matrix and peaking at intermediate angles. Then, we can see that the signal emitted by the $50 \mathrm{~nm}$ precipitate embedded right under the surface is much lower than when the same precipitate protrudes from the surface (figure 5 to be compared with figure 3). Figure 6 presents a comparison of the intensity of the signals reaching the detector and emitted by the precipitate, the matrix as well as the precipitate-tomatrix signal ratio, as a function of the precipitate size and as a function of the sample surface state, i.e. either chemically etched with precipitates protruding from the surface or conventionally prepared with precipitates embedded underneath the surface. Keep in mind that the higher the precipitate-to-matrix signal ratio on the detector (and hence in the diffraction pattern), the higher the chance of being able to determine the structure and orientation of the precipitate. According to figure 6 , one can see that the transmission signal intensity collected from a $40 \mathrm{~nm}$ precipitate protruding from the surface is as high as the backscatter signal intensity collected from a $100 \mathrm{~nm}$ precipitate embedded under the surface of a flat sample. Alternatively, the signal intensity collected from a precipitate protruding from the surface is higher by a factor of 2, 4.5 and 10 for a 100,50 and $20 \mathrm{~nm}$ precipitate, respectively. Finally, a $50 \mathrm{~nm}$ precipitate sitting on the surface produces a precipitate-to-matrix signal ratio on the detector of $40 \%$, while it is only $8 \%$ with a conventional EBSD sample. This increase in precipitate signal and precipitate-to-matrix signal ratio is the key to the success of the present technique. Next to the simulation, an experiment was also conducted with the objective of indexing precipitates in the same material but with a flat, conventional EBSD sample. Among the $50+$ precipitates that were tested, ranging in size from 50 to $100 \mathrm{~nm}$, none of them could be indexed because the contributions from precipitates to diffraction patterns were largely absent or way too faint for analysis. A typical example on a $100 \mathrm{~nm}$ precipitate is presented in figure 7 where, unlike in figure 2, no new Kikuchi bands associated to the precipitate could be identified. Besides the nature of the generated diffraction signal (transmission vs backscatter), additional effects might make it more difficult to obtain indexable patterns from precipitates embedded under the surface. One of them might be the fact that precipitates are always cut to 
some extent in flat samples, resulting in smaller volumes for precipitates of the same size in comparison to when precipitates protrude from the surface.
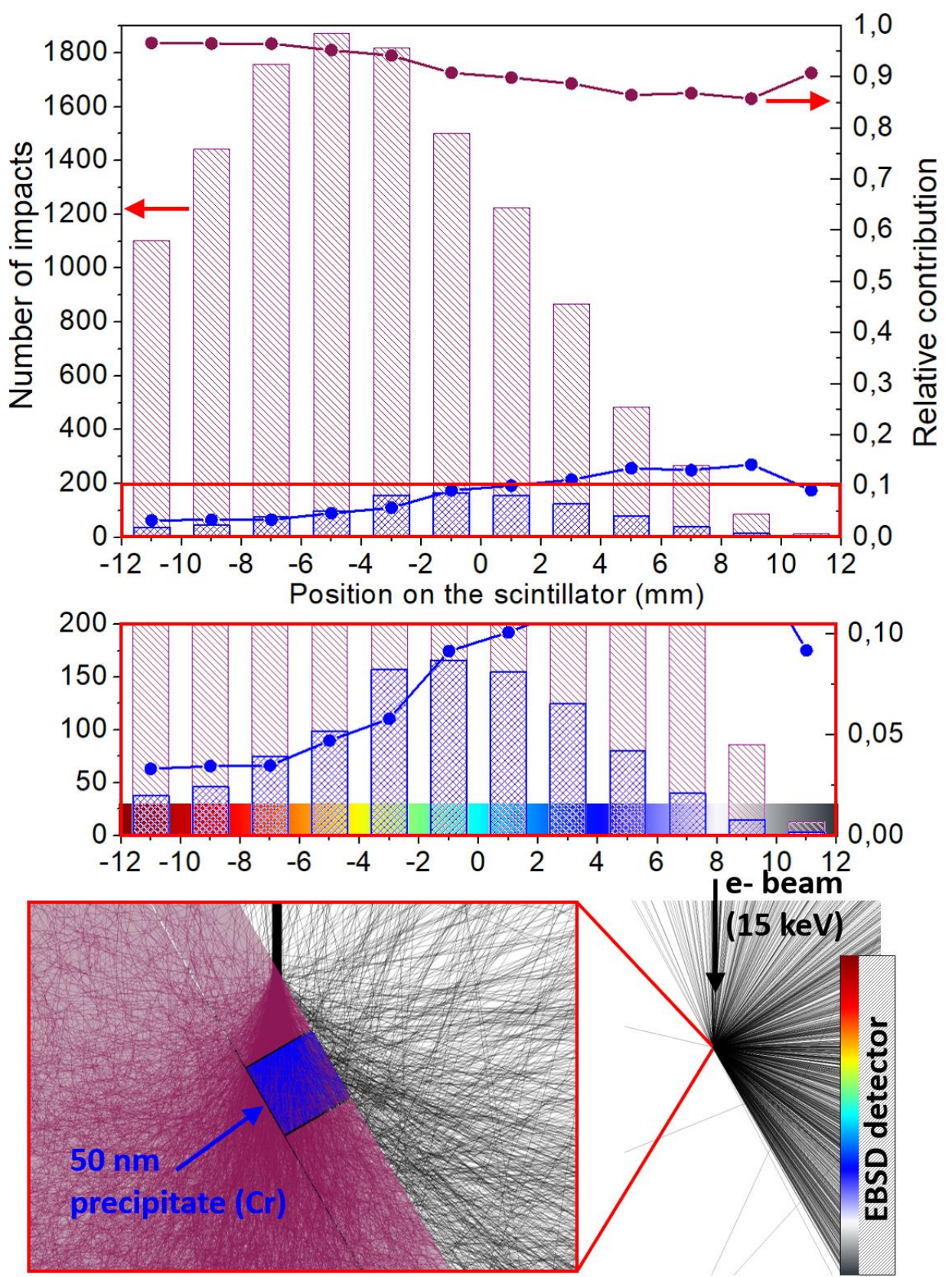

(one column figure, color) Figure 5: Result of a Monte Carlo simulation (CASINO [Drouin 2007]) showing the landing position of electrons and their relative intensity on an EBSD detector as a function of the material from which they escape, either the precipitate in blue or the matrix in purple. Unlike in figure 3, the precipitate is embedded just underneath the surface and generates a backscatter signal. See caption of figure 3 for more information on the simulation. 

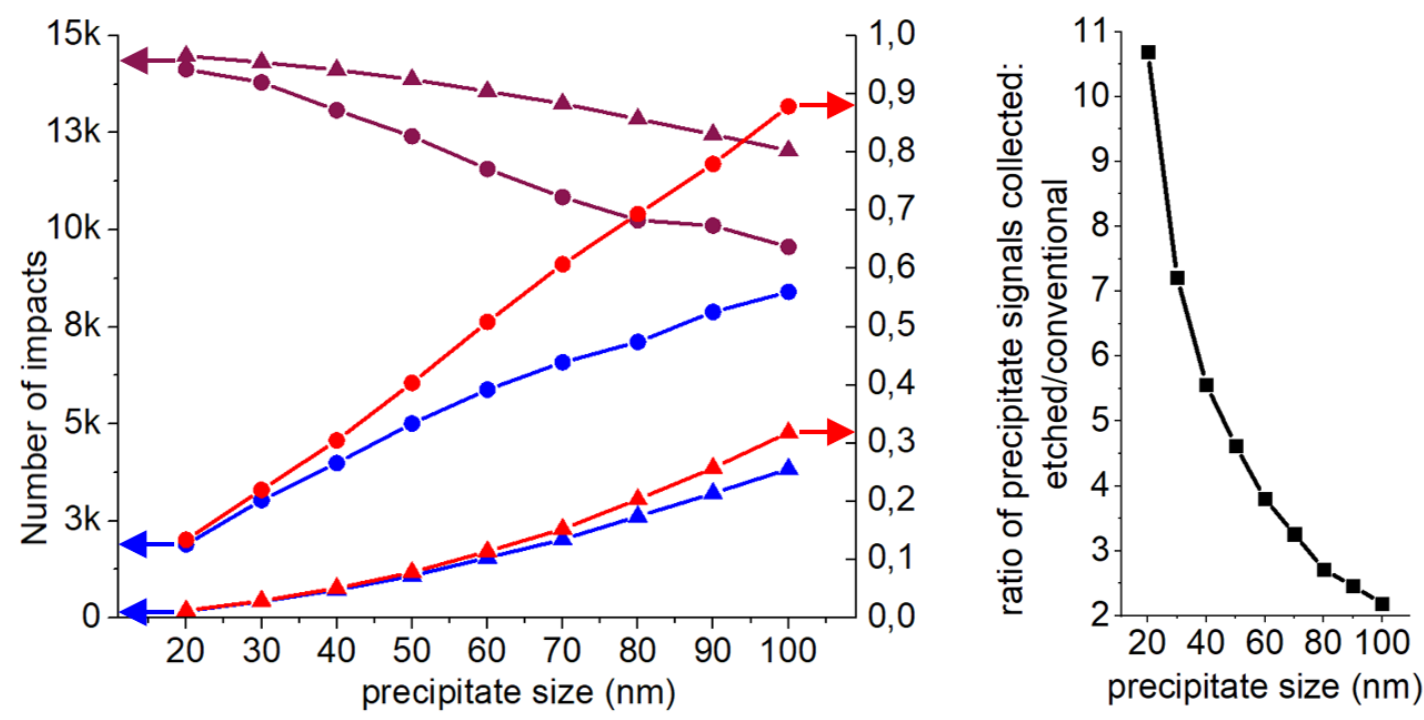

- intensity of the signal emitted by the matrix material and reaching the detector

- intensity of the signal emitted by the precipitate and reaching the detector

- ratio of precipitate-to-matrix signal intensities on the detector

(one column figure, color) Figure 6: (left) Results of a Monte Carlo simulation (CASINO [Drouin 2007]) showing the relative precipitate and matrix signal intensity on the EBSD detector as a function of the sample surface finish and the precipitate size. Triangles represent the intensity of the signal collected from a flat sample, while spheres represent the intensity of the signal collected from a selectively etched sample surface. (right) Ratio of the precipitate signal intensities reaching the detector when comparing a selectively etched surface with a conventionally prepared surface.

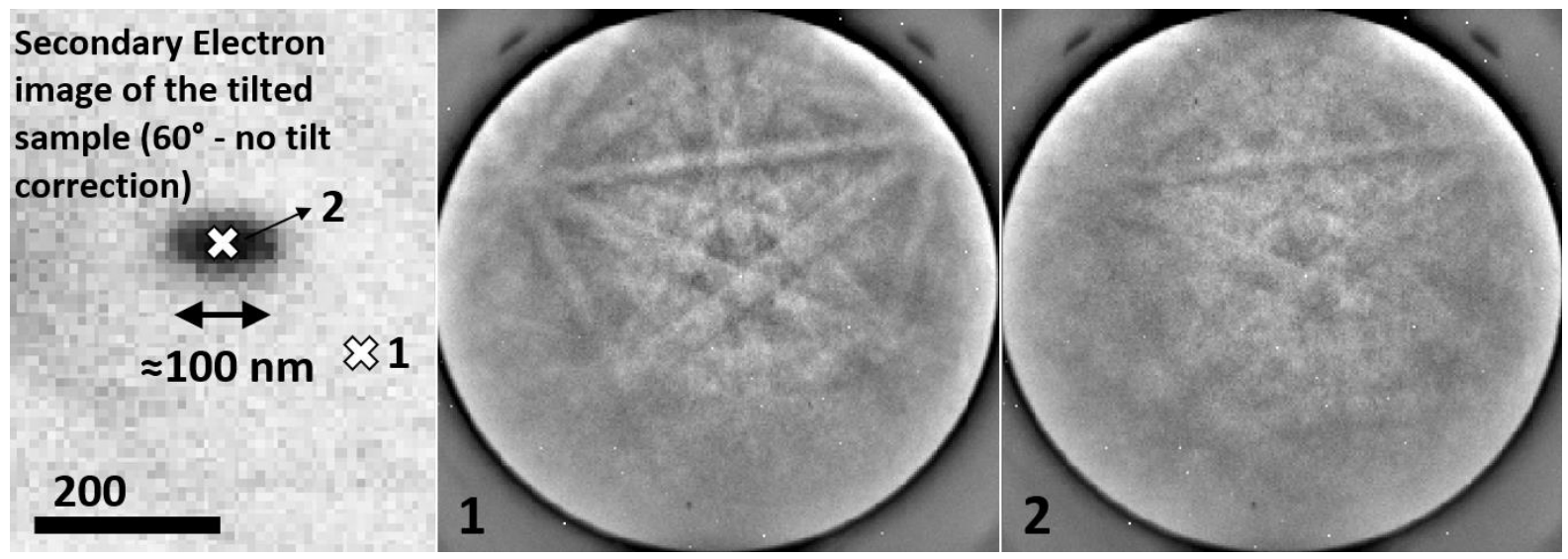

(one column figure) Figure 7: Tentative of structure and orientation analysis of a $100 \mathrm{~nm}$ Cr precipitate in a $\mathrm{Cu}$ matrix by EBSD with a conventionally prepared EBSD sample (i.e. flat, with precipitates embedded underneath the surface). Here the analysis was unsuccessful because beside the matrix, no bands from the precipitate could be reliably identified. The surface is tilted by $60^{\circ}$ and the incident energy is $15 \mathrm{keV}$. 


\section{Conclusion}

A new SEM technique in-between transmission and backscatter diffraction, easy to implement and requiring only basic EBSD hardware, is proposed to retrieve the structure and orientation of nanometer-sized precipitates directly from bulk samples. It allows analysis of precipitates whose size are well below the spatial resolution limit of the EBSD technique and that require usually thin lamella extraction and TEM analysis. The technique relies on a selective chemical etch of the matrix material of a sample, combined with the large tilt characteristic of the EBSD technique. Thanks to the surface topography induced by the selective etching, precipitates protrude from the sample surface and emit transmission diffraction signals instead of backscatter diffraction signals, even though the sample remains massive. The transmission nature of the signal generated was first deduced from the characteristic experimental angular distribution of that signal, and then confirmed thanks to a Monte Carlo simulation in which the signals generated by a protruding and a free-standing precipitate are compared.

With this technique, the structures and orientation relationships of several precipitates down to $30 \mathrm{~nm}$ were successfully retrieved in a bulk $\mathrm{Cu} / \mathrm{Cr}$ sample produced by Laser Powder Bed Fusion additive manufacturing and subsequently aged. On the contrary, when the same material was conventionally prepared for EBSD analysis (i.e. flat), out of the 50+ precipitates that we tested in the $50-100 \mathrm{~nm}$ range, none could be indexed because the diffraction patterns contained information almost exclusively from the matrix.

Using Monte Carlo simulations, it was confirmed that the intensity of the transmission signals collected from nanometer-sized precipitates are much higher than the backscatter signals collected from the same precipitates embedded right under the surface of a flat sample. According to the simulation, the transmission signal collected from a $40 \mathrm{~nm}$ precipitate protruding from the surface is as high as the backscatter signal collected from a $100 \mathrm{~nm}$ precipitate embedded below the surface. Most importantly, the precipitate-to-matrix signal ratio on the detector is increased, which is the reason for the success of this new technique: the signal ratio is $40 \%$ for a $50 \mathrm{~nm}$ precipitate protruding from the surface, while it is only $8 \%$ for the same precipitate embedded under the surface of a flat sample.

The smallest precipitate size that can be analyzed with this new technique is likely a function of atomic number (higher atomic numbers should allow analysis of even smaller precipitates), microscope performance (beam size vs beam current and stability) and diffraction 
pattern post-processing routine. In particular, we used a very basic post-processing routine. It simply consisted in subtracting a matrix pattern from a pattern recorded with the beam incident on precipitates, in order to isolate the contributions from precipitates and make indexing easier and more reliable. With a more sophisticated routine it should be possible to index patterns from even smaller precipitates and emitting lower intensity signals.

Besides diffraction, the topography induced by the chemical selective etch also makes the imaging of precipitates very easy with secondary electrons in comparison to a flat sample, and also allows a more accurate determination of the precipitate size and shape.

Finally, a great advantage of this methodology, in comparison to thin lamella extraction, is that large areas can be explored, which is very convenient when microstructural features in a material range from the nanometer scale to several tens of micrometers, as is often the case in modern materials. Besides, this technique is not limited to nano-scale precipitates and could very well be applied for structure and orientation analysis of phases at grain boundaries for example, provided that the right etching solution is identified.

\section{Acknowledgements}

This work was supported by the by the Flanders Innovation \& Entrepreneurship agency (Agentschap Innoveren en Ondernemen (VLAIO)) in the framework of the Strategic Basic Research (SBO) project MultiMet [grant number 150010]

\section{Data Availability}

The website: Materials Today Data Availability guidelines cannot be accessed at the moment, so that will wait.

\section{References}

[Bhattacharya 2019] A. Bhattacharya, C. M. Parish, J. Henry, Y. Katoh, High throughput crystal structure and composition mapping of crystalline nanoprecipitates in alloys by transmission Kikuchi diffraction and analytical electron microscopy, Ultramicroscopy 202 (2019) 33-43 
[Breen 2014] A. J. Breen, K. Y. Xie, M. P. Moody, B. Gault, H.-W. Yen, C. C. Wong, J. M. Cairney, S. P. Ringer, Resolving the Morphology of Niobium Carbonitride Nano-Precipitates in Steel Using Atom Probe Tomography, Microscopy and Microanalysis 20, (2014) 1100-1110

[Brodu 2017-1] E. Brodu, E. Bouzy, Depth resolution dependence on sample thickness and incident energy in on-axis transmission Kikuchi diffraction in scanning electron microscope (SEM), Microscopy and Microanalysis 23 (2017) 1096-1106

[Brodu 2017-2] E. Brodu, E. Bouzy, J-J. Fundenberger, Diffraction contrast dependence on sample thickness and incident energy in on-axis Transmission Kikuchi Diffraction in SEM, Ultramicroscopy 181 (2017) 123-133

[Brodusch 2013] N. Brodusch, H. Demers, R. Gauvin, Nanometres-resolution Kikuchi patterns from materials science specimens with transmission electron forward scatter diffraction in the scanning electron microscope, Journal of Microscopy 250 (2013) 1-14

[Chbihi 2012] A. Chbihi, X. Sauvage, D. Blavette, Atomic scale investigation of $\mathrm{Cr}$ precipitation in copper, Acta Materialia 60 (2012) 4575-4585

[Drouin 2007] D. Drouin, A. R. Couture, D. Joly, X. Tastet, V. Aimez, R. Gauvin, CASINO V2.42 - A fast and easy-to-use modeling tool for scanning electron microscopy and microanalysis users, Scanning 29 (2007) 92-101

[Fuji 2000] T. Fujii, H. Nakazawa, M. Kato, U. Dahmen, Crystallography and morphology of nanosized $\mathrm{Cr}$ particles in a $\mathrm{Cu}-0.2 \% \mathrm{Cr}$ alloy, Acta Materialia 48 (2000), 1033-1045

[Gautam 2013] A. S. Gautam, J. M. Howe, A method to predict the orientation relationship, interface planes and morphology between a crystalline precipitate and matrix: part II application, Philosophical Magazine 93 (2003) 3472-3490

[Guo 2002] Z. Guo, W. Sha, Quantification of precipitation hardening and evolution of precipitates, Materials Transactions 43 (2002) 1273-1282

[Hall 1972] M. G. Hall, H. I. Aaronson, K. R. Kinsma, The structure of nearly coherent fcc/bcc boundaries in a Cu-Cr alloy, Surface Science 31 (1972) 257-274

[Handbook] ASM Specialty Handbook - Copper and Copper Alloys, Edited by J. R. Davis, 2001, Materials Park, USA 
[Hodoroaba 2016] V-D. Hodoroaba, S. Rades, T. Salge, J. Mielke, E. Ortel, R. Schmidt, Characterisation of nanoparticles by means of high-resolution SEM/EDS in transmission mode, IOP Conf. Series: Materials Science and Engineering 109 (2016) 012006

[Jiao 2017] Z. B. Jiao, J. H. Luan, W. Guo, J. D. Poplawsky, C. T. Liu, Atom-probe study of $\mathrm{Cu}$ and $\mathrm{NiAl}$ nanoscale precipitation and interfacial segregation in a nanoparticle-strengthened steel, Materials Research Letters 5, (2017) 562-568

[Jozwik 2018] I. Jozwik, A. Strojny-Nedza, M. Chmielewski, K. Pietrzak, L. Kurpaska, S. Nosewicz, High resolution SEM characterization of nano-precipitates in ODS steels, Microscopy Research and Techniques 81 (2018) 502-508

[Keller 2012] R. R. Keller, R. H. Geiss, Transmission EBSD from $10 \mathrm{~nm}$ domains in a scanning electron microscope, Journal of Microscopy 245 (2012) 245-251

[Liu 2018] J. Liu, M. Hou, H. Yang, H. Xie, C. Yang, J. Zhang, Q. Feng, L. Wang, L. Meng, H. Wang, In-situ TEM study of the dynamic interactions between dislocations and precipitates in a Cu-Cr-Zr alloy, Journal of Alloys and Compounds 765 (2018) 560-568

[Liu 2019] J. Liu, S. Lozano-Perez, A. J. Wilkinson, C. R.M. Grovenor, On the depth resolution of transmission Kikuchi diffraction (TKD) analysis, Ultramicroscopy 205 (2019) 5-12

[Luo 1994] C. P. Luo, U. Dahmen, K. H. Westmacott, Morphology and crystallography of Cr precipitates in a Cu-0.33 wt\% Cr alloy, Acta Metallurgica et Materialia 42 (1994) 1923-1932

[Luo 1998] C.P. Luo, U. Dahmen, Interface structure of faceted lath-shaped Cr precipitates in a Cu-0.33 wt\% Cr alloy, Acta Materialia 46 (1998) 2063-2081

[Nolze 2004] G. Nolze, Characterization of the fcc/bcc orientation relationship by EBSD using pole figures and variants, Zeitschrift für Metallkunde 95 (2004) 744-755

[Shi 2012] R. Shi, N. Ma, Y. Wang, Predicting equilibrium shape of precipitates as function of coherency state, Acta Materialia 60 (2012) 4172-4184

[Sneddon 2016] G. C. Sneddon, P. W. Trimby, J. M. Cairney, Transmission Kikuchi diffraction in a scanning electron microscope: A review, Materials Science and Engineering R 110 (2016) $1-12$ 
[Song 2017] L. L. Song, S. Liu, X. Mao, A new method for fast statistical measurement of interfacial misfit strain around nano-scale semicoherent particles, RSC Advances 7 (2017) 28506-28512

[Suzuki 2013] S. Suzuki, Features of transmission EBSD and its application, Journal of Microscopy 65 (2013) 1254-1263

[Verbeken 2009] K. Verbeken, L. Barbé, D. Raabe, Evaluation of the Crystallographic Orientation Relationships between FCC and BCC Phases in TRIP Steels, ISIJ International 49 (2009) 1601-1609 